TITLE:

\title{
Modeling and emergence of flapping flight of butterfly based on experimental measurements
}

\section{AUTHOR(S):}

Senda, Kei; Obara, Takuya; Kitamura, Masahiko; Nishikata, Tomomi; Hirai, Norio; lima, Makoto; Yokoyama, Naoto

\section{CITATION:}

Senda, Kei ... [et al]. Modeling and emergence of flapping flight of butterfly based on experimental measurements. Robotics and Autonomous Systems 2012, 60(5): 670-678

\section{ISSUE DATE:}

2012-05

URL:

http://hdl.handle.net/2433/157348

\section{RIGHT:}

(C) 2011 Elsevier B.V.; This is not the published version. Please cite only the published version.; この論文は出版社版でありません。引用の際に は出版社版をご確認ご利用ください。 


\title{
Modeling and emergence of flapping flight of butterfly based on experimental measurements
}

\author{
Kei Senda ${ }^{\mathrm{a}, *}$, Takuya Obara ${ }^{\mathrm{b}}$, Masahiko Kitamura ${ }^{\mathrm{b}}$, Tomomi Nishikata ${ }^{\mathrm{b}}$, \\ Norio Hirai ${ }^{\mathrm{c}}$, Makoto Iima ${ }^{\mathrm{d}}$, Naoto Yokoyama ${ }^{\mathrm{a}}$ \\ ${ }^{a}$ Kyoto University, Yoshida-Honmachi, Sakyo-ku, Kyoto, 606-8501, JAPAN \\ ${ }^{b}$ Kanazawa University, Kanazawa, Ishikawa 920-1192, JAPAN \\ ${ }^{c}$ Osaka Prefecture University, JAPAN \\ ${ }^{d}$ Hiroshima University, JAPAN
}

\begin{abstract}
The objective of this paper is to clarify the principle of stabilization in flapping-of-wings flight of a butterfly, which is rhythmic and cyclic motion. For this purpose, a dynamics model of a butterfly is derived by Lagrange's method, where the butterfly is considered as a rigid multi-body system. For the aerodynamic forces, a panel method is applied. Validity of the mathematical models is shown by agreement of the numerical result with the measured data. Then, periodic orbits of flapping-of-wings flights are searched in order to fly the butterfly models. Almost periodic orbits are obtained, but the model in the searched flapping-of-wings flight is unstable. This research, then, studies how the wake-induced flow and the flexibly torsional wings effect on the flight stability. Numerical simulations demonstrate that both the wake-induced flow and the flexible torsion reduces the flight instability. Because the obtained periodic flapping-of-wings flight is unstable, a feedback control system is designed, and a stable flight is realized.
\end{abstract}

Keywords: modeling, flapping flight, butterfly, experimental measurements

\footnotetext{
*Principal corresponding author

Email addresses: senda@kuaero.kyoto-u.ac.jp (Kei Senda), n_hirai@envi.osakafu-u.ac.jp (Norio Hirai), makoto@mis.hiroshima-u.ac.jp (Makoto Iima), yokoyama@kuaero.kyoto-u.ac.jp (Naoto Yokoyama)
} 


\section{Introduction}

Butterflies can maintain desired flapping-of-wings flights against environmental uncertainties and variations, e.g. gust, weight gain or loss, etc., using their adaptation-capability. Such an adaptive function is considered to emerge from the interaction of the nervous system, body, and environment. We call the adaptive motor function "Mobiligence."

The flapping flight of butterfly is an example of Mobiligence in which the environment is the generated flowfield. Actually, it essentially has the same structure with other Mobiligence subjects, e.g., to emerge the flapping flight from the interaction of the nervous system, body, and environment. From the viewpoint, this study discusses the principle to emerge the stable flapping flight of butterfly, which is rhythmical periodic motion.

There are many unknown points for the stable flight, e.g. "how the butterfly moves," "how the control is realized," etc. Concretely, this study investigates the following two issues by the biological analysis through experimental observations of living butterflies and by the systems engineering or synthetic approach. This paper summarizes a part of study results obtained by a research project based on this approach. References $[1,2,3,4,5,6,7,8]$ reports some studies in detail.

An experimental system with a low-speed wind tunnel is constructed to measure the motion and aerodynamic forces of actual butterflies quantitatively. A 3D mathematical model is constructed by using a panel method for aerodynamics formulation in order to analyze the stability of free-flying butterflies and so on. Its validity and accuracy are examined by comparing with the obtained experimental data. A periodic trajectory of flapping-ofwings flight is searched in order to fly the constructed butterfly model. Using the constructed numerical simulator and the obtained periodic trajectory, it is shown that the free-vortices in the wakes provides a type of stabilization effect. In addition, wing torsion caused by structural flexibility is also introduced to the model and its effect on the flapping-of-wings flight is examined. But the obtained trajectory is still unstable, and the butterfly model goes down after a few flapping periods. Therefore, a feedback control system is discussed to realize a stable flight. A designed controller stabilizes the flapping-of-wings flight, where the fully nonlinear model is controlled by a sampled-data linear controller.

The rest of this paper is organized as follows. Section 2 organizes flapping mechanism of butterfly using anatomical observation and knowledge of mus- 
culoskeltal system. Section 3 shows observation experiments and measured results. Section 4 explains modeling of butterfly, in which a butterfly is considered as a rigid multi-body system, and aerodynamic forces are formulated by a panel method. Its numerical results are compared with the measured data, and their good agreement justifies the validity and accuracy of the mathematical model. In section 5 , a periodic trajectory of flapping-of-wings flight is searched by using the constructed numerical simulator, and an almost periodic trajectory is obtained. Wing torsion caused by structural flexibility is also introduced to the model and its effect on the flapping-of-wings flight is examined. Numerical simulations show the extension of the flying period considering them. Meanings of this stabilization effect is discussed, which can be considered as a common principle of mobiligence. Because the obtained periodic flapping-of-wings flight is unstable, a feedback control system is designed in section 6, and a stable flight is realized. Finally, concluding remarks are given in section 7 .

\section{Flapping Mechanism of Butterfly}

\subsection{Outline of Flapping Mechanism}

Fig. 1 schematically illustrates a flapping mechanism of insects that is powered by indirect muscles [9]. The wings are connected to the thorax by lever mechanisms, where the wings have been evolved from exoskeleton. The end of the wing link is connected by a hinge with the lateral suctum in the thorax. The wing link is also connected by another hinge near the previous hinge with the thoral wall in the thorax. When the suctum lifts up, the principle of leverage moves the wing down. The horizontal muscles known as dorsal longitudinal muscles tends to contract and increase the curvature of the suctum to which the muscles are connected to the rear and the front. There are vertical muscles known as the dorsoventral muscles running from the roof of the thorax to the floor. Contraction of the dorsoventral muscles pulls the suctum down. While relaxation of the dorsoventral muscles results in the dorsal longitudinal muscles' contraction and the suctum's lifting up. This flapping mechanism can be considered as almost one degree-of-freedom vibrating mechanism. A butterfly contracts the dorsoventral muscles periodically to vibrate the mechanism for flapping of the wings. According to experimental observation in latter section, each butterfly has an almost constant flapping eigenfrequency. It may be because to vibrate sympathetically at eigenfrequency of the mechanism realizes efficient flapping motion [10]. 


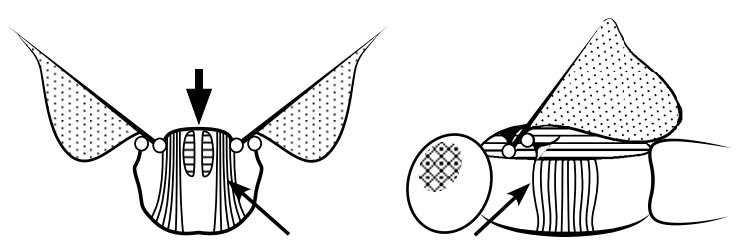

Dorsoventral muscle contraction

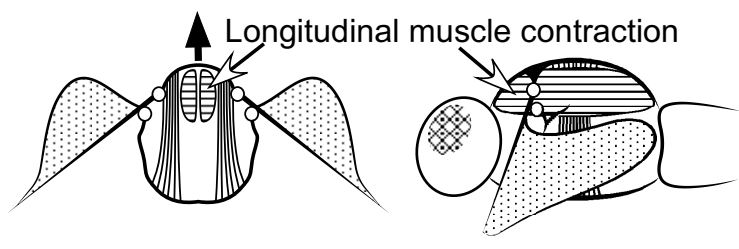

Figure 1: Indirect muscle system driving wing mechanism (left: thorax cross section, right: thorax longitudinal section)

As suggested in the anatomical observation below, the other direct muscles may add slight motions, which are in the extent of modification to the basic vibration.

\subsection{Anatimical Observation of Butterfly}

To clarify the possible flight movements, morphology of thoracic muscles of a danaid butterfly, Parantica sita niphonica is investigated using the microXCT scanner, Comscantecno ScanXmate-A080S. The X-ray tube voltage, the tube current, and the minimum resolution (i.e. slice width and pitch) are adjusted $70 \mathrm{kV}, 90 \mathrm{~mA}$, and $18 \mu \mathrm{m}$, respectively. Three-dimensional images are obtained by the software, Analyze. Fig. 2 shows examples of 3D images reconstructed with XCT images as wings are in horizontal position. The following investigation uses XCT images of the spacemen whose wings are in horizontal, top, and bottom positions.

As a result, the above-mentioned major indirect flight muscles including dorsal longitudinal muscles and dorso-ventral muscles of this species are identified. Some direct flight muscles such as basalar muscles and subalar muscles are attached below the forewings via basalare and subalare, respectively.

As known in other butterflies, Parantica sita niphonica basically uses indirect muscles for upstroke and downstroke and uses direct muscles for forward and rearward inclination of forewings. Therefore, it is reasonable to assume that living Parantica sita niphonica can generate the motions applied to a mathematical model in the latter section. 


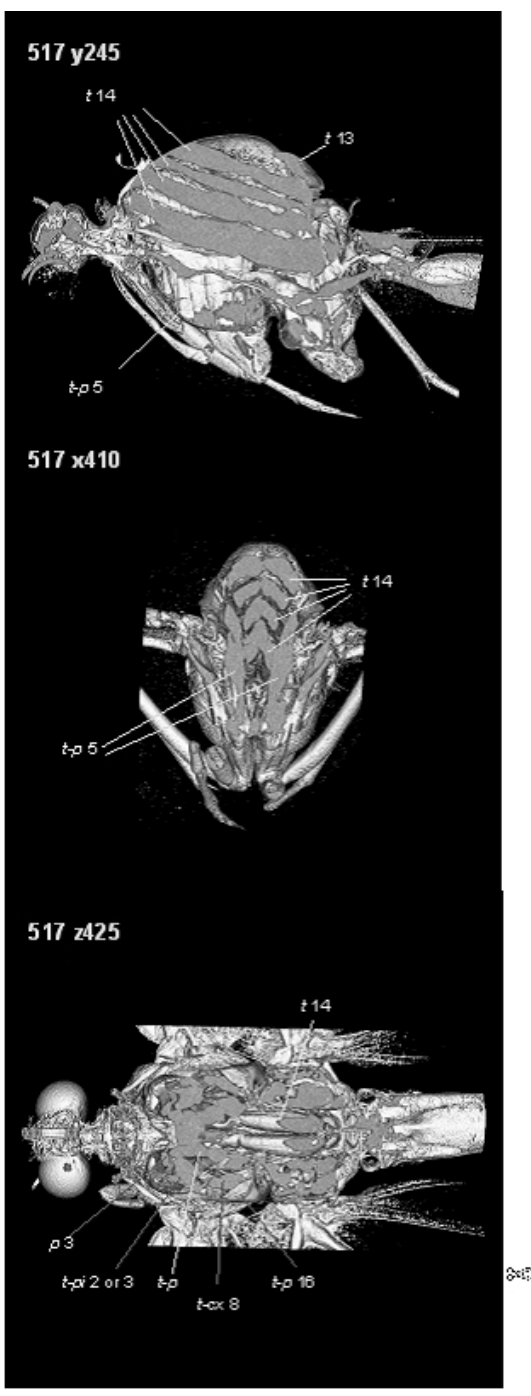

Figure 2: 3D images reconstructed with micro-XCT images of Parantica sita niphonica 


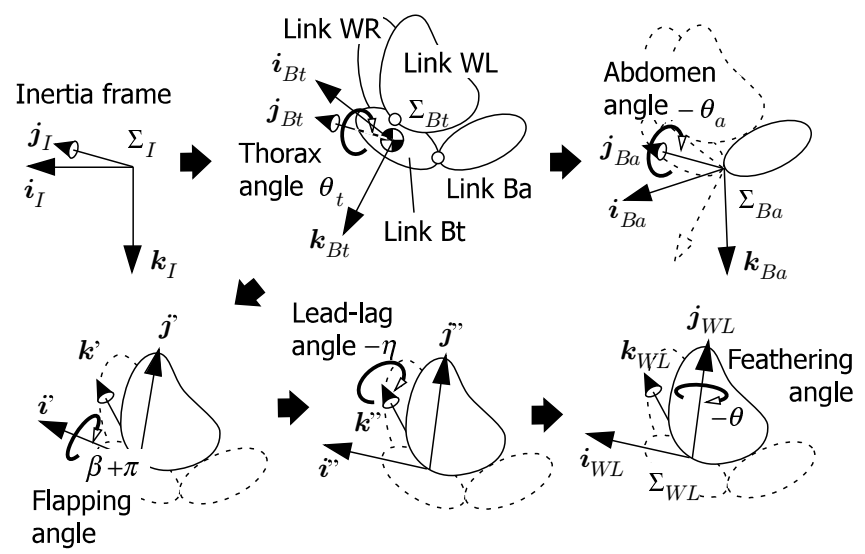

Figure 3: Frames and coordinates for modeling of a butterfly

\section{Models of Butterfly}

\subsection{Dynamics Model as Rigid Multi-body System}

A butterfly is modeled by a rigid multi-body system as well as typical robot modeling, which is simple dynamics modeling. For more precise modeling, it will be desirable to consider a flexible multi-body system because experimental observations have found that butterfly wings are elastically deformed by aerodynamic forces.

The butterfly model is a multi-body system with 4 links as shown in Fig. 3, which is composed of the thorax Bt considered as the main body, the abdomen $\mathrm{Ba}$, the left wings WL, and the right wings WR. Both the model and its motions are supposed to be symmetrical. A pair of fore and hind wings on each side is modeled by a plate as shown in Fig. 3. The joint between the thorax and wing has 3 rotational degree-of-freedom (DOF) and the joint between the thorax and the abdomen has 1 DOF.

The following Lagrangian equations of motion are obtained:

$$
\boldsymbol{M} \ddot{\boldsymbol{\theta}}+\dot{\boldsymbol{M}} \dot{\boldsymbol{\theta}}-\frac{1}{2} \frac{\partial}{\partial \boldsymbol{\theta}}(\dot{\boldsymbol{\theta}} \boldsymbol{M} \dot{\boldsymbol{\theta}})+\frac{\partial V}{\partial \boldsymbol{\theta}}=\boldsymbol{\tau}
$$

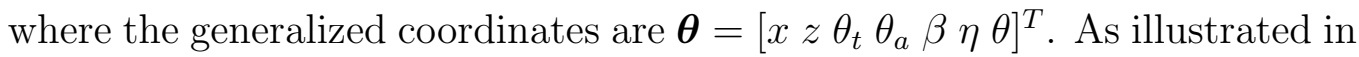
Fig. $3, x, z$, and $\theta_{t}$ are the $\mathrm{x}, \mathrm{y}$-positions and the attitude angle of the thorax, $\theta_{a}$ the abdomen angle, $\beta$ the flapping angle in up-down direction, $\eta$ the leadlag angle, and $\theta$ the feathering angle representing a torsion angle. The $\boldsymbol{M}$ and $V$ are the inertia matrix and the gravitation potential energy, respectively. 
The vector $\boldsymbol{\tau}=\boldsymbol{\tau}_{d}+\boldsymbol{\tau}_{\text {control }}$ is the generalized forces corresponding to $\boldsymbol{\theta}$, where $\boldsymbol{\tau}_{d}$ and $\boldsymbol{\tau}_{\text {control }}$ are the aerodynamic torque and the joint control torque, respectively, developed in the following sections.

\subsection{Aerodynamics Models}

In the flight of the butterfly, there is a feature in which the flapping frequency is small [10] as well as small Reynolds number. Moreover, the flow to the wings is not steady because of the flapping motion, whereas the flow to a cruising fixed-wing aircraft is steady. Wing-tip vortices greatly affect the aerodynamic characteristics because a butterfly has flat wings of a small aspect ratio. In addition, the flapping motion generates very strong wingtip vortices. The wing-tip vortices may combine to the free-vortices leaving from the trailing edge, make complex structures, and greatly influence the aerodynamic characteristics.

Therefore, this study models the aerodynamics of a flapping-of-wings butterfly using a panel method [11]. Vortex ring element panels are set on wing surfaces and panels with constant strengths of vortices are shed into wakes. Unknown strengths of vortices are determined so as to satisfy the boundary condition of no normal flow across the wing surfaces. The free wake model sheds the panels fulfilling the Kutta condition at trailing edge and the panels move with local stream velocity. This free wake model enables to contain the influence of the unsteady wakes. As a result, this study made a panel method model $[3,4,5]$.

A lumped-vortex method model is introduced in this paper to discuss the effect of unsteady wakes by comparing with the panel method. The lumpedvortex method model considers only the vortex ring panels on wing surfaces, removing the panels in wakes from the panel method model. However, the model reffered as the lumped-vortex method in this paper additionally considers the characteristic flapping-of-wings effects [3], where each effect is modeled separately. This study has also developed other aerodynamic models $[3,4,5]$, whereas they are not mentioned in this paper

\section{Experiments and Simulations}

\subsection{Outline of Experimental System}

Verification of the models and parameter setting require fundamental data of flapping-of-wings motions and aerodynamic forces. For this purpose, an experimental system with a low-speed wind tunnel is constructed and a wind 


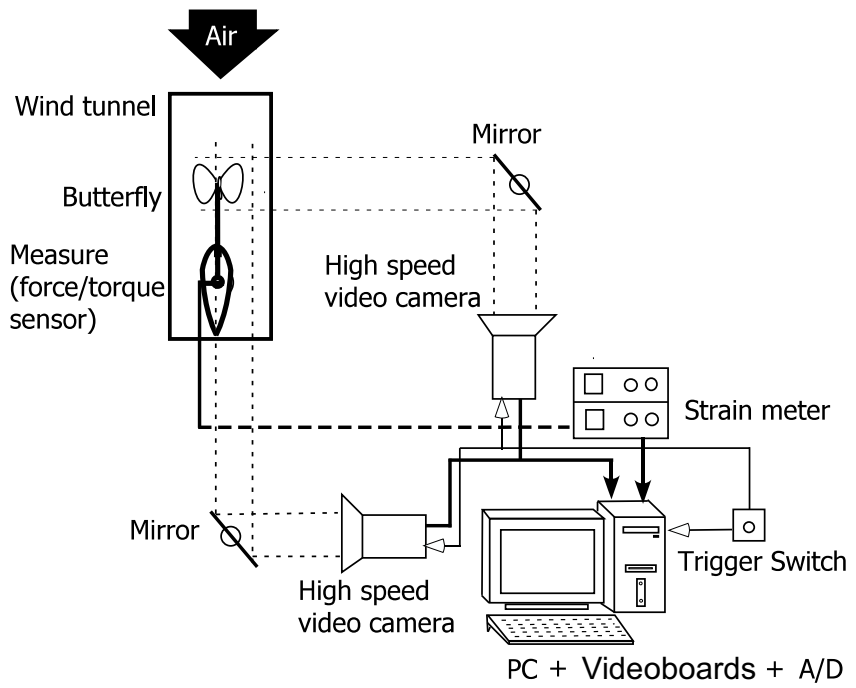

Figure 4: Experimental setup

tunnel experiment is conducted using actual butterflies [4], scientific name Parantica sita niphonica, which is similar to Danaus in the US. It is $0.24 \mathrm{~g}$ weight and its half wing span is $50 \mathrm{~mm}$ long. Specifications of the butterfly are listed in Ref [2].

The simultaneous measurement system is constructed as illustrated in Fig. 4, which measures butterfly's motion and the forces applied to the butterfly using an optical measurement system and a force measurement system. The butterfly is gummed up on the back of the thorax to the tip of the measure, i.e. the force/torque sensor. The measure with the butterfly is put into the wind tunnel, the flapping-of-wings motion in the flow is captured as a video image, and the state vector $\boldsymbol{\theta}$ is measured using the video images. Simultaneously, the forces applied to the butterfly, i.e. lift $L, \operatorname{drag} D$ and pitching moment $M$, are sensed by the measure. There is a smoke wire for airflow visualization.

According to calibration experiments, the maximum measuring error in $L$ and $D$ is $0.001[\mathrm{~N}]$ for a range of $\pm 0.02[\mathrm{~N}]$, and that in $M$ is $0.04 \times 10^{-3}[\mathrm{Nm}]$ for $\pm 0.6 \times 10^{-3}[\mathrm{Nm}]$. The cutoff frequency of the measure is $50[\mathrm{~Hz}]$ for noise reduction. The maximum error in position measurements using the video images is $0.13 \times 10^{-3}[\mathrm{~m}]$ in each direction, which is caused by the resolution of images: $0.260 \times 10^{-3}[\mathrm{~m} / \mathrm{pixel}]$. Based on this error, the maximum error 


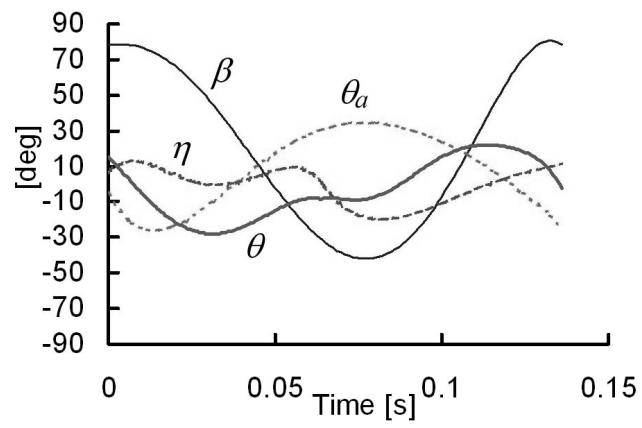

(a) Motion

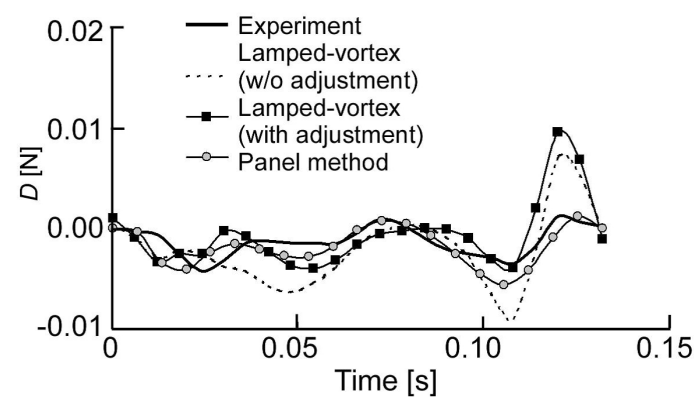

(c) Drag

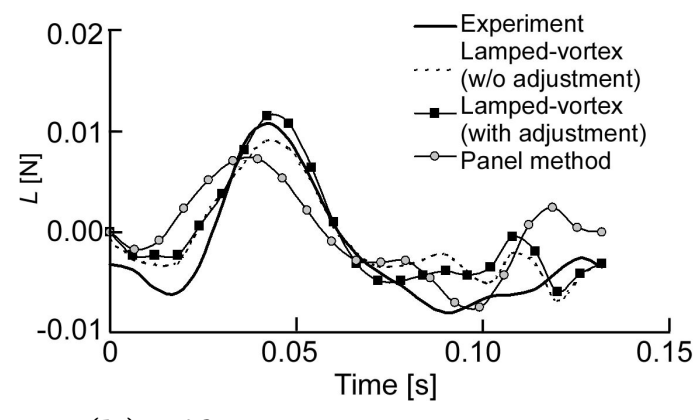

(b) Lift

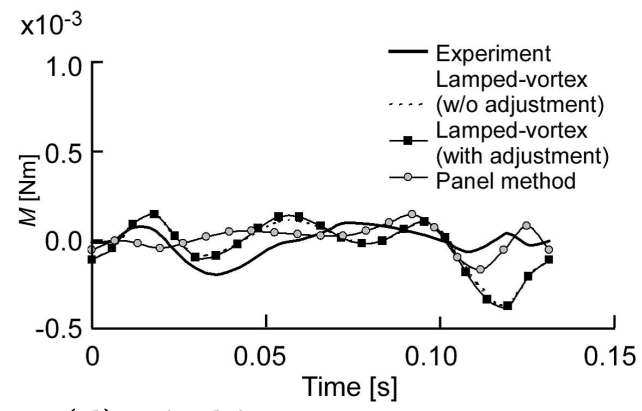

(d) Pitching moment

Figure 5: Aerodynamic forces of lumped-vortex method and panel method

in the abdomen angle is $0.37^{\circ}$ for motion measurement. The smoke is made of the propylene glycol by smoke-wire method. According to an evaluation method in [12], the tracers in smoke follow-up accurately the air-flow.

\subsection{Experimental Observation of Motion and Force}

An experiment for Parantica sita niphonica is conducted under a condition that the mainstream is $1.64 \mathrm{~m} / \mathrm{s}$, the thoracic positions $x=z=0 \mathrm{~m}$, and its angle $\theta_{t}=-1^{\circ}$. Fig. 5 (a) shows the generalized coordinates $\boldsymbol{\theta}$ of an experimental result measured by the above optical measurement system. Figs. 5 (b)-(d) illustrate the measured forces at the tip of the measure, i.e. the lift $L$, the drag $D$, and the force moment $M$. The butterfly repeats this cyclic motion in a period of 0.136 seconds when it continues the periodic flapping-of-wings motion. Measured aerodynamic forces are also cyclic during the periodic flapping-of-wings motion.

The flapping angle $\beta$ is a cosine-like curve whose maximum and minimum are $81^{\circ}$ and $-45^{\circ}$. The left and right wings overlaps perfectly at the 
maximum position. The abdomen angle $\theta_{a}$ is also a sinusoidal curve, but is out of phase to the flapping angle $\beta$. When the butterfly varies amplitude, it changes the minimum of $\beta$ mainly and the maximum marginally. The fixed butterfly swings the abdomen unnaturally in a larger amplitude than butterflies flying freely. Even if $\beta$ or $\theta_{a}$ changes the amplitude depending on the flying condition and/or the individual, they keep similar smooth cosine curves. Lead-lag angle $\eta$ has a vibration mode whose frequency is twice as large as $\beta$. Feathering angle $\theta$ seems to be varied passively in small by the aerodynamic forces. Fry et al. [13] similarly observes fly's flapping-of-wings motion.

As can be seen in Fig. 5 (a), the feathering angle $\theta$ varies approximately between a range of $\pm 30^{\circ}$ through a flapping period. This fluctuation of the $\theta$ is considered as a result of a passive wing torsion caused by its structural flexibility since butterflies cannot actively twist their wings in a large angle because of the flapping mechanism. In a latter section, the passive wing torsion is introduced to the model, and its effect on the flapping-of-wings flight stability is evaluated.

\subsection{Verification of Mathematical Models}

The $\boldsymbol{\theta}$ obtained from the experiment and its derivatives $\dot{\boldsymbol{\theta}}$ and $\ddot{\boldsymbol{\theta}}$ are substituted into the mathematical models of the butterfly that is fixed on the back of the thorax to the tip of the measure. Its aerodynamic forces are illustrated in Figs. 5 (b)-(d). The aerodynamic models are the lumpedvortex method with the characteristic flapping effects and the panel method. Validity of the model can be evaluated by comparing the simulation results with the experimental data.

For the lumped-vortex method, two results are represented. One is the result using the parameters suitable for the past experimental result of Parantica sita niphonica under a condition that the mainstream is $1.0 \mathrm{~m} / \mathrm{s}$ and the thoracic angle $\theta_{t}=31^{\circ}$. The other is the result after adjusting the parameter to be suitable for the present experiment. The lumped-vortex method model with the characteristic flapping effects has many adjustable parameters. Hence the model with parameters for the present experiment obtains better agreement with the measured result in comparison with the one for the other experiment. On the other hand, the model for the other experiment shows some error at the top or the bottom of flapping angle. This means that the lumped-vortex method model must use the parameters suitable for instantaneous condition, e.g. the angle of attack, the flow velocity to the 


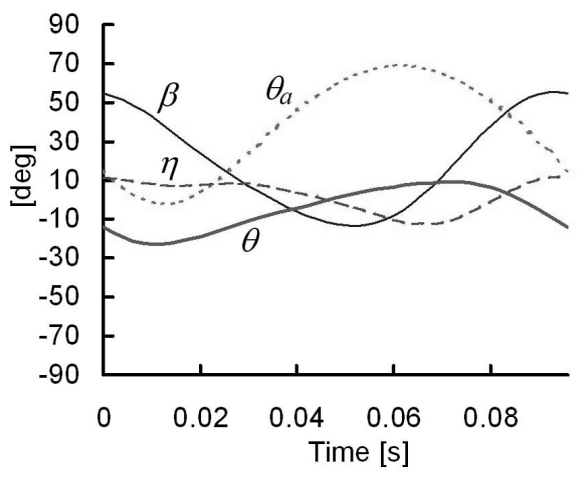

(a) Motion

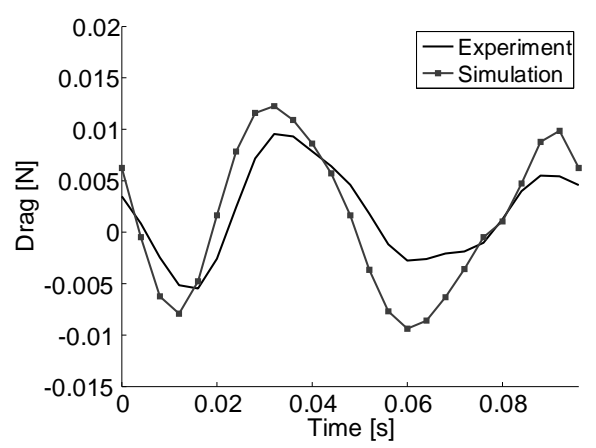

(c) Drag

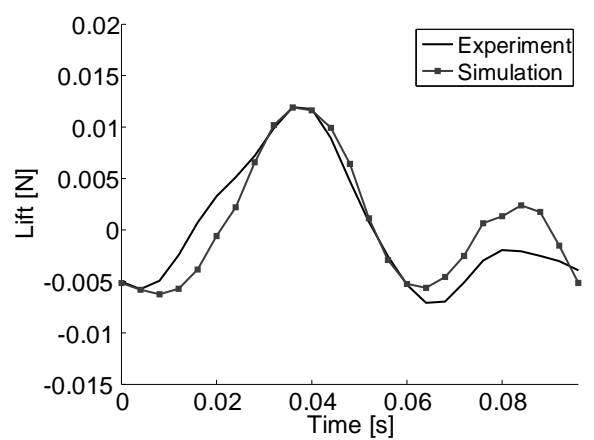

(b) Lift

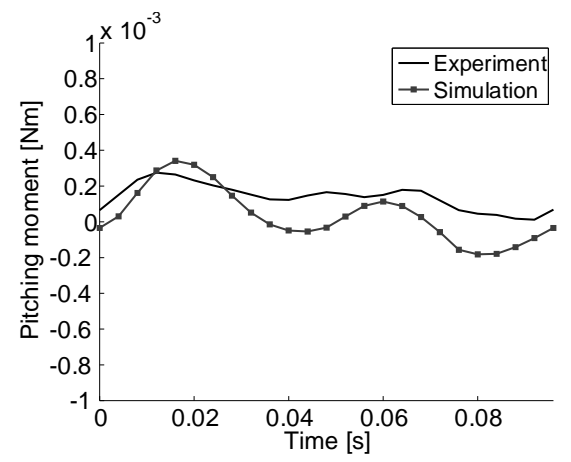

(d) Pitching moment

Figure 6: Verification of panel method by aerodynamic forces

wings, etc. The lumped-vortex method model is not necessarily suitable for a free-flying butterfly because the flow is unsteady and the condition always varies.

On the other hand, the panel method can calculate the aerodynamic forces with the same degree of accuracy for any experiment. Fig. 6 shows the experimental result of Parantica sita niphonica under a condition that the mainstream is $1.54 \mathrm{~m} / \mathrm{s}$ and the thoracic angle $\theta_{t}=28.1^{\circ}$, and the numerical result by the panel method. The panel method model always agrees well with experimental results by considering unsteady flow induced by wakes.

Left figures in Fig. 7 show that the flow around the wings visualized by a smoke wire. They are side view images of every 4 frames shot in 250 FPS, i.e. the interval of the images is $0.016 \mathrm{~s}$.

Referring the inertial frame $\Sigma_{I}$ in Fig. 3, the origin of thorax frame $\Sigma_{B t}$ is at the origin of $\Sigma_{I}$, the mainstream flows in $-\boldsymbol{i}_{I}$ direction , and the sagittal 
plane of the butterfly is on $\boldsymbol{i}_{I^{-}} \boldsymbol{k}_{I}$ plane. In this case, the smoke wire is placed upstream of the butterfly, in $-0.044 \boldsymbol{j}_{I}[\mathrm{~m}]$, and parallel to $\boldsymbol{k}_{I}$, where the length of each wing is almost $0.05[\mathrm{~m}]$. A trail of smoke from a point makes a streak line, and the smoke from the whole smoke wire forms a sheet. In case without butterfly, the sheet-like smoke forms a plane that parallels to $\boldsymbol{i}_{I^{-}} \boldsymbol{k}_{I}$ plane.

The brightness of smoke has a positive correlation with the density of tracers because the whole area is lighted uniformly and the images are taken from $-\boldsymbol{j}_{I}$ direction. The brightness of the plane of sheet-like smoke without butterfly is almost constant everywhere in an image. In case with a butterfly, the sheet-like smoke bends out-of-plane direction because butterfly flapping induces three dimensional flow with $\boldsymbol{j}_{I}$ velocity. The flapping-induced vortex structure can be estimated qualitatively from the brightness of the smoke in an image because the tracer density becomes lower at larger induced velocity. From the experimental result, it is understood that the complex flowfield is formed by the flapping-induced free vortex in wakes.

In numerical simulations of the panel method, the velocity at any point can be obtained by Biot-Savart law, which calculates the velocity induced by each vortex element. In right figures, small arrows at lattice points denote the local velocities relative to mainstream calculated by the panel method. In depth direction, the arrows are illustrated simultaneously from $-0.12[\mathrm{~m}]$ to $0[\mathrm{~m}]$. In the figures, more long arrows appear at a region with larger induced velocity. These figures are suitable to compare with the experimental images qualitatively. This numerical simulation captures the unstedy flowfield of the experiment. For reference, the lumped-vortex method model does not have any abilities to describe the complex unsteady flow.

Viscosity effect should be considered in the model because of the low Reynolds number $R e \simeq 10^{3}$ for the butterfly. However, both computational and experimental evaluations show that the viscous drag is negligible amount with approximately $0.5 \%$ of the maximum drag. Compressibility is not taken into account because of low speed. It is observed in Figure 8 by the smoke wire visualization that the flow passes along wing surfaces without separation during wingbeat. The panel method model might result in good agreement with the real flow, where the model is based on potential flow theorem assuming nonviscous and incompressive flow without separation.

Motion of a free-flying butterfly is measured by using the experimental system of Figure 4. Figure 9 (a) and (b), respectively, show a period of measured entire butterfly motion in periodic free-flight and the numerical 

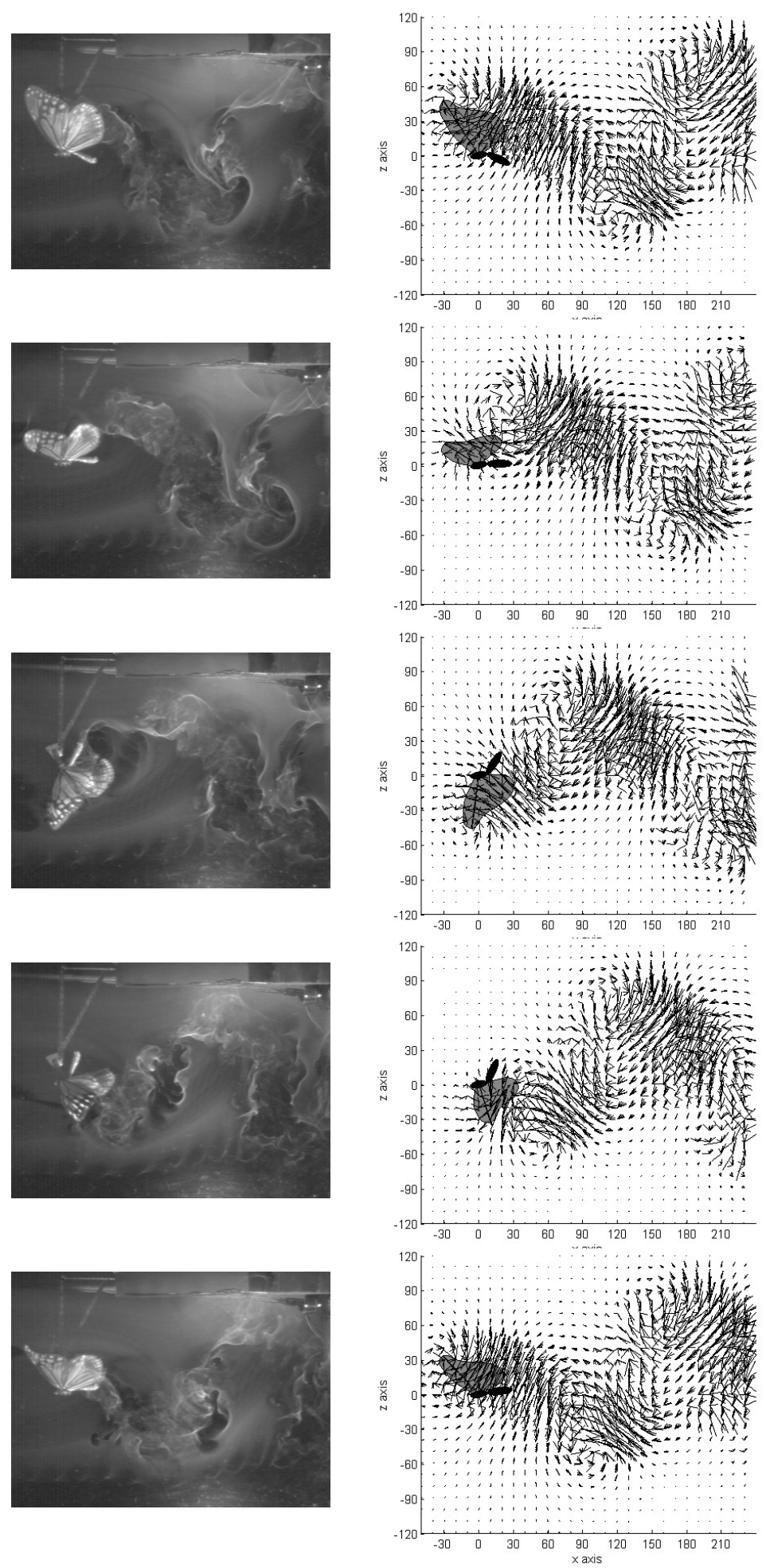

Figure 7: Comparison of flow fields of experimental visualization and numerical simulation by panel method every $0.036 \mathrm{~s} .\left(V=1.0 \mathrm{~m} / \mathrm{s}, \theta_{t}=-10^{\circ}\right)$ 


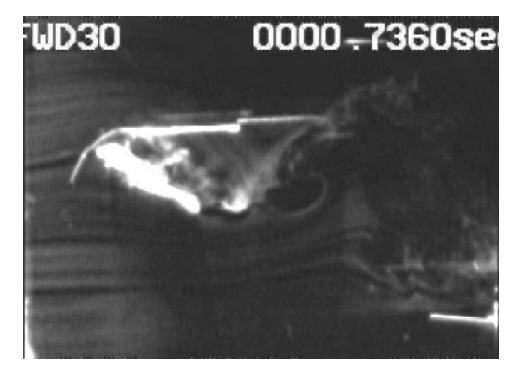

Figure 8: Visualized flow around wing during downstroke. $(V=1 \mathrm{~m} / \mathrm{s})$

result when entire motion is calculated by the obtained model using the measured joint trajectory. The numerical result duplicates the free-flight, even though the final thorax angles have some difference. The obtained model is the most accurate among models constructed by the present authors, i.e. simple method [1], the lumped-vortex method considering the characteristic flapping-of-wings effects [2], and the panel method model without freevortices in wakes, because the other existing models cannot duplicate the free-flight.

\section{Periodic Flapping Flight}

Even when the measured joint trajectory of an actual butterfly is used, the entire free-flying motion of numerical model is different from the actual butterfly. This result shows that the model cannot reproduce the actual butterfly perfectly, whereas the model duplicates the free-flight. Hence, a flapping joint trajectory is searched below for a periodic free-flight of the numerical model. Using the obtained model and trajectory, effects of freevortices in wakes and structural flexibility of wings are discussed.

\subsection{Trajectory Search}

The periodic flapping-of-wings flight is defined as "all other than $x$ in state vector $\boldsymbol{\theta}_{f}$ and $\dot{\boldsymbol{\theta}}_{f}$ after a flapping-of-wings period $T$ agree with initial value $\boldsymbol{\theta}_{s}$ and $\boldsymbol{\theta}_{s}$."

Joint angle trajectories are described by Fourier series for cyclic motions. Fourier parameters and initial conditions are considered as learning parameters of vector $\boldsymbol{w}$ to find a trajectory of the periodic flapping-of-wings flight. In order to reduce computation efforts, the number of parameters is cut down based on the knowledge from the experiments and observation. 


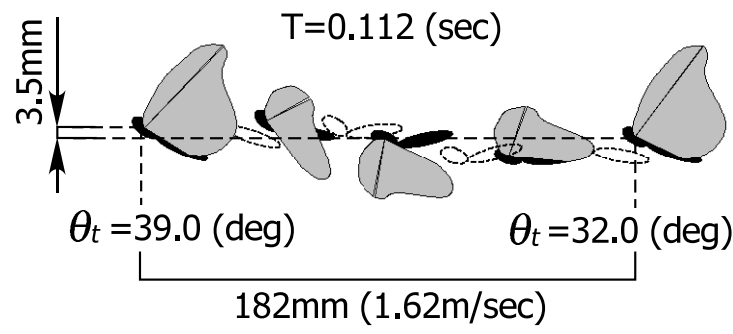

(a) experimental measurement

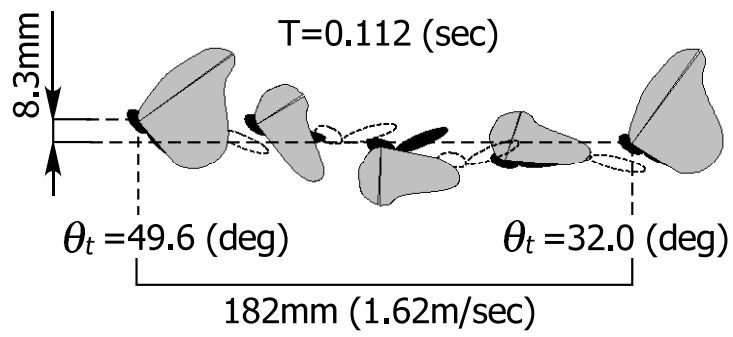

(b) numerical simulation

Figure 9: Measured free-flying of flapping butterfly and numerical simulation using measured joint motion

The following cost function $J$ is used for the learning evaluation:

$$
\begin{aligned}
J= & \sum_{i} Q_{i}\left(\theta_{f i}-\theta_{s i}\right)^{2} \\
& +R\left\{E\left(\boldsymbol{\theta}_{f}, \dot{\boldsymbol{\theta}}_{f}\right)-E\left(\boldsymbol{\theta}_{s}, \dot{\boldsymbol{\theta}}_{s}\right)+\sum_{j} \int_{0}^{T} \tau_{j} d \theta_{j}\right\}
\end{aligned}
$$

where $E(\boldsymbol{\theta}, \dot{\boldsymbol{\theta}})=T(\boldsymbol{\theta}, \dot{\boldsymbol{\theta}})+V(\boldsymbol{\theta})$ is the mechanical energy with the kinetic energy $T(\boldsymbol{\theta}, \dot{\boldsymbol{\theta}})$ and the potential energy $V(\boldsymbol{\theta})$. The $\theta_{j}$ and $\tau_{j}$ are the angle and the torque of joint $j$. The $Q_{i}$ and $R$ are evaluation weights. The suffix $i$ denotes $z$ or $\theta_{t}$ or $\dot{x}$ or $\dot{z}$ or $\dot{\theta}_{t}$. The suffix $j$ denotes $\theta_{a}$ or $\beta$ or $\eta$ or $\theta$. The first term of Eq. (2) evaluates the periodicity of motion by quadratic form of error between boundaries. The second term of Eq. (2) is mechanical energy loss of the period. One obtains the most efficient flapping-of-wings flight for a mathematical model by minimizing the cost function. The obtained trajectories are evaluated by comparing with experimental results. A gradient method finds the above-mentioned learning parameters such that the obtained trajectory satisfies the definition of the periodic flapping-of-wings flight. 


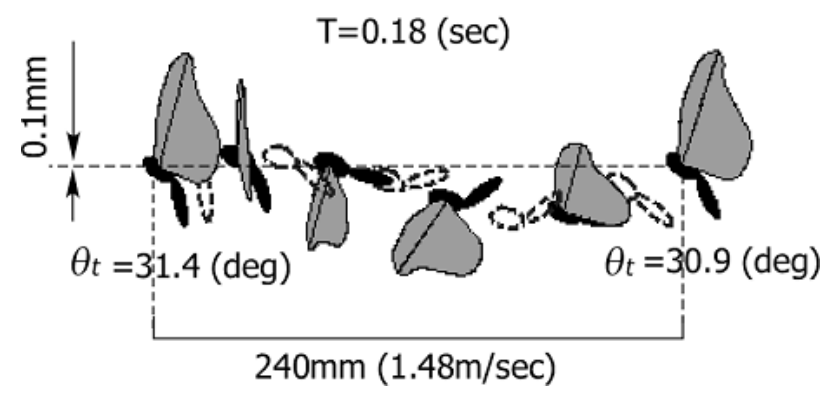

(a) model with free-vortices in wakes

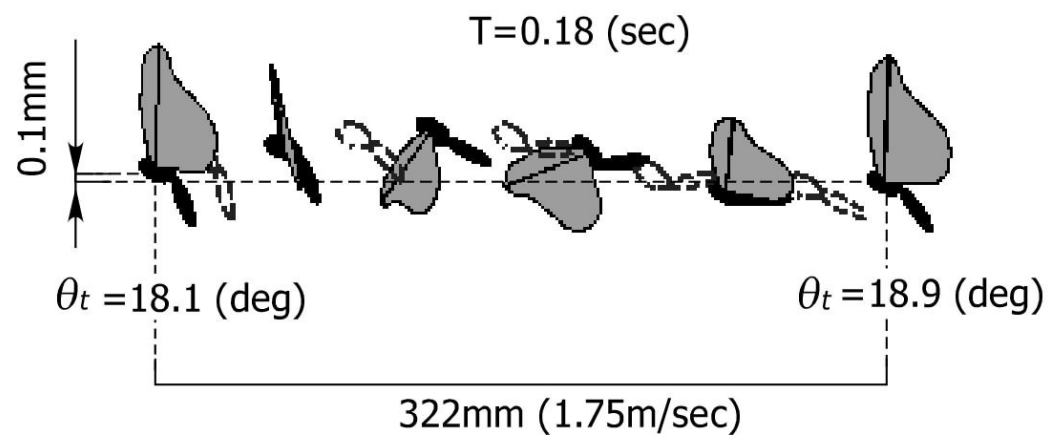

(b) model without free-vortices in wakes

Figure 10: Almost periodic flapping-of-wings flight of models

The panel method model can obtain almost periodic trajectories, though they do not perfectly correspond to the definition of the periodic flight. The obtained periodic flapping flight by the panel method model is illustrated in Fig. 10 (a).

\subsection{Stability Analysis for Periodic Trajectory}

We analyze stability of the periodically flapping flight.

A continuous system of periodically flapping butterfly is considered as

$$
\dot{\boldsymbol{x}}(t)=\boldsymbol{f}(\boldsymbol{x}(t), t)
$$

where state vector is $\boldsymbol{x}(t)=\left[\boldsymbol{\theta}^{T}(t), \dot{\boldsymbol{\theta}}^{T}(t)\right]^{T}$.

Consider $\boldsymbol{x}^{0}(t)$ is a periodic trajectory with period $T$ of the continuous system of Eq. (3). The state vector of the continuous system is obtained every $T$, i.e. $t_{k}=k T(k=0,1,2, \ldots)$, and the sampled-data system is made as:

$$
\boldsymbol{x}\left(t_{k+1}\right)=\overline{\boldsymbol{f}}\left(\boldsymbol{x}\left(t_{k}\right)\right)
$$


The sampled-data system Eq. (4) is expanded by the following Taylor series about $\boldsymbol{x}^{0}$.

$$
\boldsymbol{x}\left(t_{k+1}\right)=\overline{\boldsymbol{f}}\left(\boldsymbol{x}^{0}\left(t_{k}\right)\right)+\frac{\partial \overline{\boldsymbol{f}}}{\partial \boldsymbol{x}^{T}}\left(\boldsymbol{x}\left(t_{k}\right)-\boldsymbol{x}^{0}\left(t_{k}\right)\right)+\cdots
$$

The following perturbation equation of the sampled-data system is obtained by considering $\delta \boldsymbol{x}\left(t_{k}\right)=\boldsymbol{x}\left(t_{k}\right)-\boldsymbol{x}^{0}\left(t_{k}\right)$ is very small, regarding the second and higher terms of the Taylor series are negligible, and taking account of $\boldsymbol{x}^{0}\left(t_{k+1}\right)=\overline{\boldsymbol{f}}\left(\boldsymbol{x}^{0}\left(t_{k}\right)\right)$ :

$$
\delta \boldsymbol{x}\left(t_{k+1}\right)=\frac{\partial \overline{\boldsymbol{f}}}{\partial \boldsymbol{x}^{T}} \delta \boldsymbol{x}\left(t_{k}\right) \triangleq \boldsymbol{A} \delta \boldsymbol{x}\left(t_{k}\right)
$$

The $\boldsymbol{A}$ is Jacobian matrix of $\boldsymbol{x}\left(t_{k+1}\right)$ with respect to $\boldsymbol{x}\left(t_{k}\right)$. Using this sampled-data system, the stability can be analyzed as the evolution of perturbation.

We have relations

$$
\begin{aligned}
\boldsymbol{A} & =\boldsymbol{\Xi} \boldsymbol{\Lambda} \boldsymbol{\Xi}^{-1} \\
\boldsymbol{\Xi} & =\left[\boldsymbol{\xi}_{1} \boldsymbol{\xi}_{2} \cdots \boldsymbol{\xi}_{n}\right] \\
\boldsymbol{\Lambda} & =\operatorname{diag}\left[\lambda_{1} \lambda_{2} \cdots \lambda_{n}\right]
\end{aligned}
$$

where $\lambda_{i}$ and $\boldsymbol{\xi}_{i}$ are an eigenvalue and its normalized eigenvector of Jacobian matrix $\boldsymbol{A}$, respectively. Therefore, Eq. (6) is rearranged as

$$
\delta \chi\left(t_{k+1}\right)=\Lambda \delta \chi\left(t_{k}\right)
$$

where

$$
\begin{aligned}
\boldsymbol{x} & =\boldsymbol{\Xi} \boldsymbol{\chi} \\
\boldsymbol{\chi} & =\left[\begin{array}{llll}
\chi_{1} & \chi_{2} & \cdots & \chi_{n}
\end{array}\right]^{T}
\end{aligned}
$$

and $\chi$ is the modal coordinates vector. The $\lambda_{i}$ gives the expansion rate of the perturbation $\delta \chi_{i}$ of the mode $i$ corresponding to the $\boldsymbol{\xi}_{i}$. Hence, the mode of the $i$-th eigenvector is stable for $\left|\lambda_{i}\right| \leq 1$ and unstable for $\left|\lambda_{i}\right|>1$. The unstable mode instabilizes the butterfly motion in the direction of the corresponding eigenvector. 


\subsection{Effects of Wakes}

To discuss effects of free-vortices in wakes leaving from trailing-edges, a model is constructed where free-vortices in wakes are removed from the panel method model and a steady horseshoe vortex is considered instead. This model is essentially same as the lumped-vortex method model in the previous section. This model can obtain almost periodic trajectories, too. The obtained periodic flapping flight is illustrated in Fig. 10 (b).

Because they are not perfectly periodic, the flapping flights in both Figs. 10 (a) and (b) are leaving gradually from the initial flights and destabilized. The models with and without free vortices go down after ten or more and a few periods of flapping motion, respectively.

Figs. 11 (a) and (b) show the flights started from the initial states with same small perturbations. Figs. 11 (a) and (b), respectively, are of the panel method model and the panel method model from which free-vortices in wakes are removed. Degree of instability of Fig. 11 (a) is smaller than Fig. 11 (b). In addition, the sampled-data systems are derived from the original continuous periodic systems by observing their states every flapping cycle. The highest unstable poles of the sampled-data systems of the previou section are 8.6 and 17.0 for the panel method and the panel method without free-vortices, respectively. This result gives support to the simulations above.

It is thought that the wake-induced flow of the panel method provides a type of stabilization effect. This result shows that the free-vortices in the wakes are essential for the stabilization of butterfly flights. The details are also reported in $[3,4,5,6,8]$.

The butterfly repeats the same joint motion by the controller, but the thorax motion and the flow field do not repeat a same periodic motion. Therefore, the aerodynamic forces applied to wings also vary, and this variation works as disturbance to butterfly's flight motion control. The flight motion is the entire butterfly motion that is composed of the thorax motion and the joint motion. It doesn't have function to suppress the error in thorax motion by feedback control, though the controller suppresses the error in joint motion caused by the disturbance. According to the above-mentioned stability analysis, the free-vortices in the wakes provide a type of stabilization effect. This result shows that the free-vortices induce the interaction with the feedback stabilization effect when wings in "body" exchange aerodynamic forces with "flow field." This is the feedback stabilization effect brought by this system through the dynamic characteristic of the flow field, 


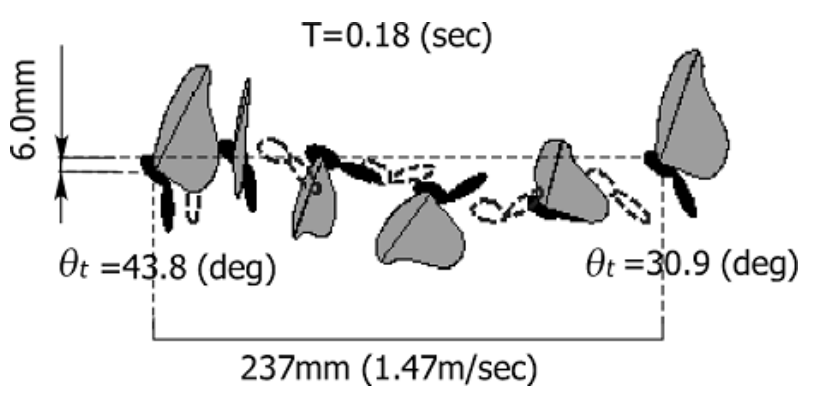

(a) slight instability of model with free-vortices in wakes

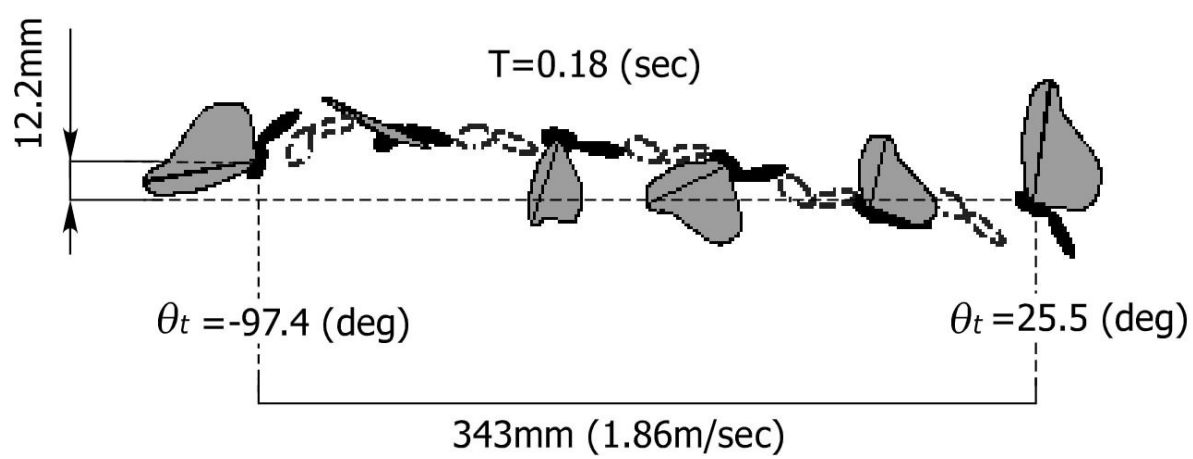

(b) instability of model without free-vortices in wakes

Figure 11: Instability of periodic flapping-of-wings flight with initial perturbation

and it can be considered as an implicit control that is a common principle of mobiligence.

\subsection{Effects of Structural Flexibility of Wings}

As discussed in Fig. 5 (a), deformation of wings caused by structural flexibility leads to large torsion. Flapping motion of the model is controlled to the desired trajectory without error in the previous section, whereas that of actual butterflies cannot be controlled as well because their wings are twisted by various unexpected disturbance. This passive torsion of wings has not been considered in the butterfly model of the above simulation. In this section, wing torsion caused by structural flexibility is introduced to the model and its effect on the flapping-of-wings flight is examined. [7]

To introduce the passive torsion to the mathematical model, the torsional rigidity of wings has been measured by using living butterflies. Torsional 


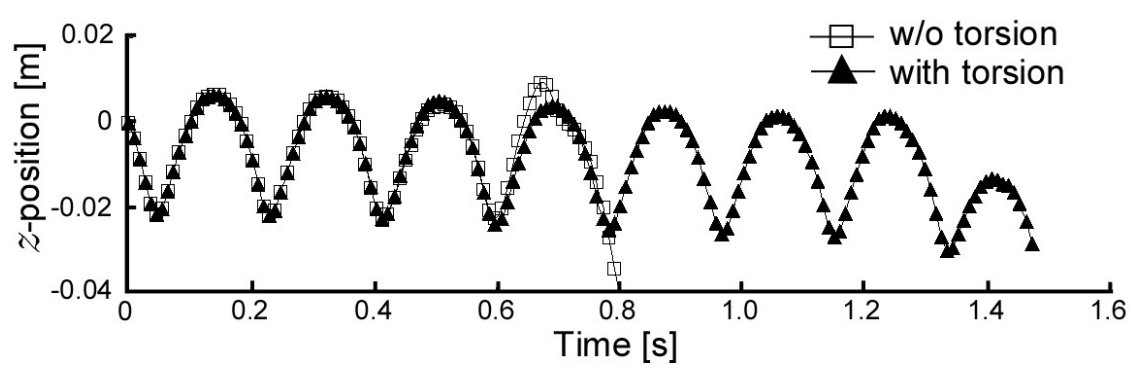

(a) Height

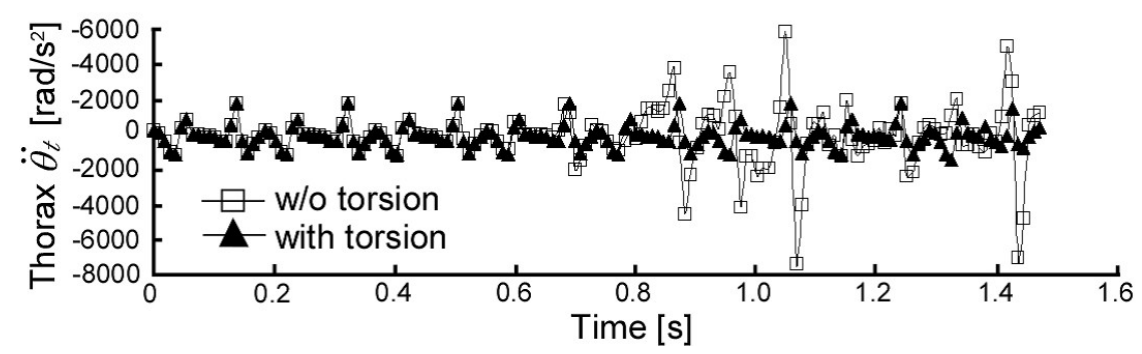

(b) Acceleration of pitching of thorax

Figure 12: Flapping-of-wings flights with/without flexible torsion

moment loads are applied to a wing of butterfly with the root of the wing fixed to the horizontal plane. The torsional rigidity of the wing is obtained by the torsion angles with respect to the torsional moment loads, where the angles are captured from camera images. The torsional rigidity of wings as an average of some living butterflies is approximately $8.0 \times 10^{-4} \mathrm{Nm} / \mathrm{rad}$.

The wing torsion caused by its structural flexibility is modeled as a singledegree-of-freedom damped oscillator, where a spring and a damper are installed in the joint at wing root. The model of wing remains as a flat plate. The controller used in the above simulation is partially modified by using PD-control so as to express the modeled passive torsion. Because it is difficult to measure damping of wings, the damping ratio is assumed to be approximately 0.7 and applied to the model.

Numerical simulations analyze how the flexible torsion changes the flight stability. Figs. 12 (a) and (b) show two trajectories of flapping-of-wings flights. By the trajectory searching, the two trajectories have been obtained using the models with and without flexibly torsional wings. It is seen that the oscillation of thorax causes the instability of the flight for the model without 
flexibly torsional wings. The flexibly torsional wings extend the stable flight for four more periods. Fig. 12 (b) shows that the oscillation of $\ddot{\theta}_{t}$ is reduced, especially when the butterfly is going down. The wing flexibly damps the pitching oscillation of thorax, where the oscillation may cause the butterfly falling. As the result, the instability of thorax reduced, and the flying period extended.

The same stabilizing effects are demonstrated in three of four other simulations by the passive wing torsion in mathematical models, where flapping motion is different in each case. Therefore, the flexible torsion may introduce the stability effect on the flapping-of-wings flight.

This is the feedback stabilization effect brought by this system through the dynamic characteristic of the body. It can be considered as a kind of preflex [14] as well as an implicit control that is a common principle of mobiligence.

\section{Feedback Control of Flapping-of-Wings Flight}

As is mentioned above, the free-vortices in wakes and the flexible torsion provide stabilization effects. But, the free-flight realized by the obtained periodic joint motion is still unstable. Hence, a feedback controller is designed to stabilize the flight. Dynamics of the periodic flapping flight is formulated as a sampled-data control system whose sampling time is the flapping period. The controller design is based on the optimal regulator for the sampled-data control system.

\subsection{Controller Design by Optimal Regulator}

The rigid multi-body model considering free-vortices in wakes and its obtained periodic joint trajectory in section 5.3 are used to formulate a sampled-data control system whose sampling time is the flapping period. The controller is then designed by using the sampled-data control system, where the state is considered as $\delta \boldsymbol{x}\left(t_{k}\right)$ for discrete time $t_{k}$ that is the difference of state $\boldsymbol{x}\left(t_{k}\right)$ from equilibrium state $\boldsymbol{x}^{0}\left(t_{k}\right)$. The input $\delta \boldsymbol{u}\left(t_{k}\right)$ is considered as the difference of Fourier parameter vectors $\boldsymbol{u}\left(t_{k}\right)$ from the periodic $\boldsymbol{u}^{0}\left(t_{k}\right)$ that represent trajectories of $\theta_{a}, \beta, \eta$, and $\theta$ in interval $\left[t_{k}, t_{k+1}\right)$. The Fourier parameter vector at time $t_{k}$ is given by $\delta \boldsymbol{u}\left(t_{k}\right)$ that is computed by the following controller.

$$
\boldsymbol{u}\left(t_{k}\right)=\boldsymbol{u}^{0}\left(t_{k}\right)+\delta \boldsymbol{u}\left(t_{k}\right)
$$



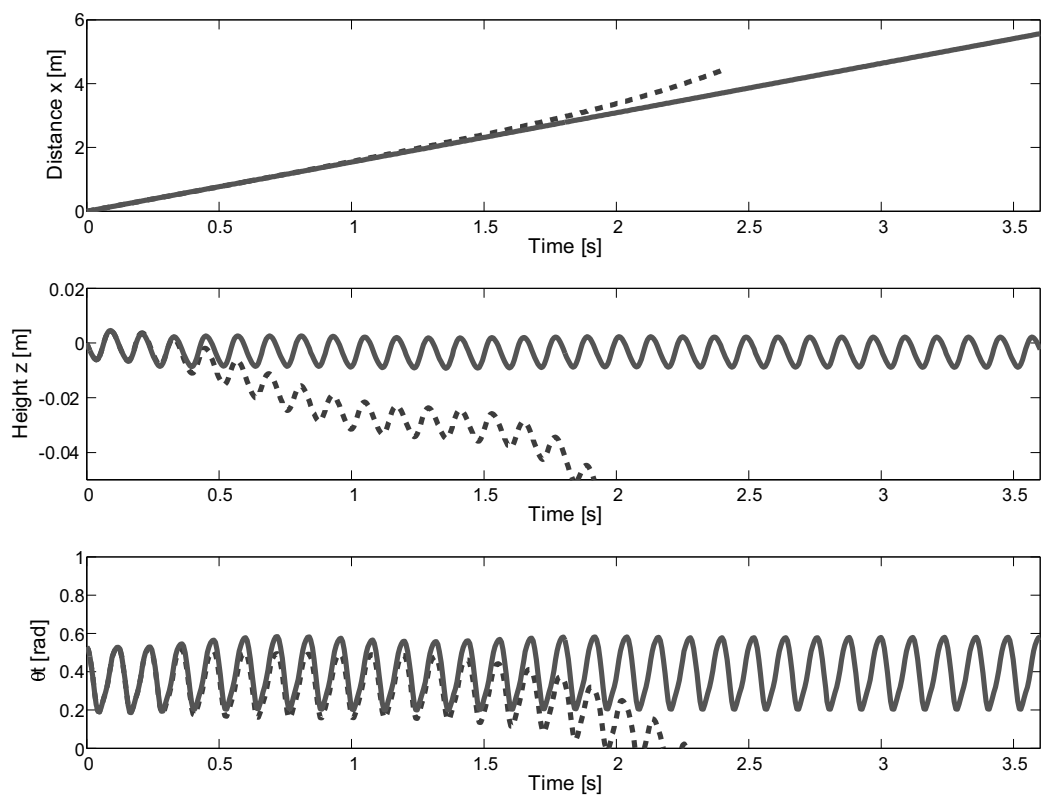

Figure 13: Free-flying motion of thorax with/without feedback control (solid line: with control , broken line: without control)

A state feedback control is designed by using the optimal regulator theory as

$$
\delta \boldsymbol{u}\left(u_{k}\right)=-\boldsymbol{K} \delta \boldsymbol{x}\left(t_{k}\right)
$$

where $\boldsymbol{K}$ is the obtained feedback gain matrix.

\subsection{Control Result}

Fig. 13 shows trajectories of flights when the designed sampled-data controller works and does not work, where the distance $x$, the height $z$, and attitude angle $\theta_{t}$. In the figure, broken lines are without the control and solid lines are with the control. The unstable trajectory describes the motion of flapping butterfly without the controller, where the trajectory is found in the previous section. The stable trajectory describes the butterfly motion with the controller. The designed sampled-data controller stabilizes the flappingof-wings flight, where the fully nonlinear model is controlled by the designed controller. More realistic control reflecting biological knowledge will be our future subject because the full state feedback control is supposed. 


\section{Concluding Remarks}

This study has analyzed the mechanism to emerge the flapping flight of butterfly considering the generated free vortices in the environment. First, flapping mechanism of butterfly has been organized by using anatomical observation and knowledge of musculoskeltal system to know the available motion of living butterflies. The experimental system with a low-speed wind tunnel has been constructed, and the motion and aerodynamic forces of actual butterflies have been measured quantitatively. Then the 3D mathematical model has been constructed. Its validity and accuracy have been examined by comparing with the obtained experimental data. Moreover, a periodic flapping flight has been realized by using the obtained model. It has clarified that the free-vortices in the wakes induced by flapping and the wing torsion caused by structural flexibility effect on the stability of flapping flight. They have been considered as the implicit controls that have been the common principle of mobiligence. But the obtained trajectory has been still unstable. The feedback control as an explicit control has been designed and the stable flight has been realized. For more realistic control reflecting biological knowledge will be our future subject.

\section{Acknowledgement}

The authors would like to express sincere thanks to Dr. Naomichi Ogihara of Keio University for the help of making XCT images of a butterfly, Prof. M. Ishii of the Osaka Prefecture Univ., and Ishikawa Insect Museum for their help making use of butterflies. A part of this work has been financially supported by a Grant-in-Aid for Scientific Research from Ministry of Education, Science, Culture, and Sports of Japan.

\section{References}

[1] K. Senda, T. Tanaka, M. Sawamoto, Measurement and numerical simulation of a flapping butterfl, in: Proceedings of 2nd International Symposium on Adaptive Motion of Animals and Machines, Kyoto, 2003, pp. $1-7, \mathrm{WeP}-\mathrm{II}-1$.

[2] K. Senda, M. Sawamoto, T. Shibahara, T. Tanaka, Study on flapping-ofwings flight of butterfly with experimental measurement, in: Proceedings of AIAA Atmospheric Flight Mechanics Conference, AIAA, Reston, Virginia, 2004, pp. 1-14, AIAA Paper 2004-5368. 
[3] K. Senda, M. Sawamoto, T. Tanaka, T. Shibahara, Analysis on control of flapping-of-wings flight of butterfly, in: Proceedings of 3rd International Symposium on Adaptive Motion of Animals and Machines, Ilmenau, Germany, 2005, pp. 1-12.

[4] K. Senda, M. Sawamoto, T. Shibahara, M. Kitamura, T. Tanaka, Study on flapping-of-wings flight of butterfly with numerical and experimental analysis, in: Proceedings of AIAA Atmospheric Flight Mechanics Conference, AIAA, Reston, Virginia, 2006, pp. 1-16, AIAA Paper 20066150 .

[5] K. Senda, M. Sawamoto, M. Kitamura, T. Tanaka, Stabilization of flapping-of-wing flight of butterfly, considering wakes, in: N. Kato, S. Kamimura (Eds.), Bio-mechanisms of Swimming and Flying, Springer, Tokyo, 2007, pp. 193-204.

[6] K. Senda, M. Sawamoto, M. Kitamura, T. Obara, Towards realization of stable flapping-of-wings flight of butterfly, in: Proceedings of 4th International Symposium on Adaptive Motion of Animals and Machines, Cleveland, Ohio, USA, 2008, pp. 62-63.

[7] K. Senda, M. Sawamoto, M. Kitamura, T. Obara, Effects of flexibly torsional wings in flapping-of-wings flight of butterfly, in: Proceedings of World Automation Congress 2008, Waikokoa, Hawaii, USA, 2008, pp. $1-6$, ISIAC-338.

[8] K. Senda, T. Obara, M. Kitamura, T. Nishikata, On flight mechanics of flapping butterfly, in: Workshop on Future Trends of Mobiligence: in IEEE International Conference on Robotics and Automation, Kobe, Japan, 2009, pp. 17-22.

[9] A. K. Brodsk, The Evolution of Insect Flight, Oxford University Press, Oxford, 1994.

[10] A. Azuma, The Biokinetics of Flying and Swimming, 2nd Edition, AIAA, Reston, Virginia, 2006.

[11] J. Katz, A. Plotkin, Low-Speed Aerodynamics, 2nd Edition, Cambridge University Press, Cambridge, 2001. 
[12] T. V. S. of Japan, Handbook of Particle Image Velocimetry, Morikita Publishing Co., Ltd., Tokyo, 2002.

[13] S. N. Fry, R. Sayaman, M. H. Dickinson, The aerodynamics of free-flight maneuvers in drosophila, Science 300 (2003) 495-498.

[14] I. E. Brown, G. E. Loeb, A reductionist approach to creating and using neuromuscular models, in: J. M. Winters, P. E. Crago (Eds.), Biomechanics and Neural Control of Posture and Movement, Springer, New York, 2000. 